\title{
Dentistry on TikTok - oh no
}

\author{
By Claire Dewshi, DCT2 in Restorative Dentistry, Swansea, UK
}

L he hashtag \#dentist has 2.4 billion views on TikTok.

The short video-sharing social media site has taken the world by storm with a mixture of snappy clips, catchy songs and an unbelievable amount of free time many of us have gained during the pandemic. The site has 1.1 billion active users ${ }^{1}$ and a huge influence over young adults in our generation.

Some of these videos can be educational - short animated videos of common dental procedures, some of it clinical - a perfectly polished prep with packed retraction cord and an immaculate impression; some of it is just light-hearted comedy - a dentist re-enacting what it's like when a goodlooking patient walks through the door.

It is impossible to escape social media as we all have mobile phones in our back pockets, and in the age where disinformation is so prevalent, can we, and should we, believe everything we see online?

\section{\#PatientPerspective}

While scrolling through the results page for 'dentist', it didn't take me long to find a video with \#lawsuit as an orthodontic patient wobbles her left lateral incisor until it falls onto her tongue, captioned: 'when you go to get your braces off, but they accidentally pull out two teeth.'2

As a young teenager about to attend for bond up, or indeed as a parent about to drive their child to the appointment, you may be concerned by the dramatic video posted with a short and uplifting soundtrack. It is important not to take these videos at face value.

On closer inspection, the lateral incisor's root looks completely resorbed and the gingiva underneath is slightly pinky-red. The 3D intraoral scan shows an arch without the upper lateral incisors, suggesting this was a planned orthodontic approach. The patient's smiling face is also reassuring that the caption was more tongue in cheek rather than real concern. In this case, jokes will certainly get a reaction, but for the unknowing public, it can be concerning if you think this is what will happen when you get your braces taken off.

\section{\#DIYDentistry}

There are of course patients who perform their own dentistry: an operculectomy, ${ }^{3}$ a midline diastema closure with small elastic bands, ${ }^{4}$ trimming an orthodontic wire with pliers ${ }^{5}$ and using a nail file to create a flat occlusal plane for the anterior teeth. ${ }^{6}$ These are just some of the many videos posted online of 'quick fixes' from people who definitely did not attend dental school.

As clinicians, we can see that this DIY dentistry shouldn't even cross a patient's mind, but if something has thousands of likes, many others will follow. In these cases, social media is potentially dangerous and can affect your long-term dental health. This is perhaps the scariest of all media crazes as we know that we will have to pick up the pieces when the homemade dental treatments fail.

\section{\#TheBenefits}

Not all videos on social media are misleading or detrimental to the dental profession.

Short animations on how wisdom teeth are surgically extracted, ${ }^{7}$ how veneers are cemented, ${ }^{8}$ and how Essix Retainers are made $^{9}$ can all be educational and help explain to patients what type of treatments we can provide. We all know a picture can paint a thousand words, and these clips can show the patient what exactly goes on when they lie back in the dental chair.

Understandably, you need to adapt to your clientele. The elderly patient asking about a new set of cobalt chromes may not appreciate a newly graduated dentist on social media, striking poses and pointing to textboxes which appear on the screen outlining the advantages of metal over an acrylic denture.

Nonetheless, videos presented in layman's terms outlining different replacement options complete with diagrams and commentary could appeal to many dental patients and supplement the verbal and written information given to them chairside.

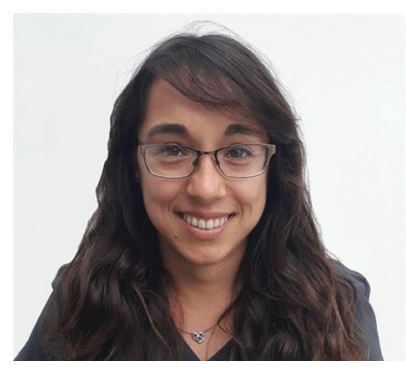

\section{\#TheFuture}

Our profession needs to adapt to the new media revolution and find a way to maintain patient safety, education and build rapport. Social media can be a powerful advertising tool, and when harnessed correctly, could aid communication with our patients.

It is important to take some of these videos with a pinch of salt and remember that anyone can take a picture and write a caption. It is our duty to fight this disinformation when we can, be it at face-to-face appointments or even on our own social media pages.

Undoubtedly, the social media algorithm has already read everything I have typed here today and will spin me down a spiral of new dental videos. Although I look forward to what it has to offer, I think I'm ready to set my social media timer to 30 minutes a day.

\section{References}

1. Wallaroo. Tik Tok Statistics - Updated June 2021. 14 June 2021. Available at: https://wallaroomedia. com/blog/social-media/tiktok-statistics/ (accessed September 2021).

2. TikTok. Lol Lawsuit. Available at: https:// www.tiktok.com/@adriennebundy/ video/6986005323572169989?lang=en\&is_copy url=1\&is_from_webapp=v1 (accessed September 2021).

3. TikTok. I'm my own dentist. Available at: https://www.tiktok.com/@baileyniblett/ video/6915233794370866438?lang=en\&is_copy_ url=1\&is_from_webapp=v1 (accessed September 2021).

4. TikTok. Closing my tooth gap in 3 days. Available at: https://www.tiktok.com/@diana..nova/ video/6960010926548389125?lang=en\&is_copy_ url=1\&is_from_webapp=v1 (accessed September 2021).

5. TikTok. At home dentistry. Available at: https://www.tiktok.com/@j.jellooo_/ video/6886916894092266758?is_copy_url=1\&is_from_ webapp=v1\&item_id=6886916894092266758\#/@j. jellooo_/video/6886916894092266758 (accessed September 2021).

6. TikTok. I hope I make dentists cringe. Available at: https://www.tiktok.com/@uyharafrancis/ video/6879497240763747586?lang=en\&is_copy_ url=1\&is_from_webapp=v1 (accessed September 2021).

7. TikTok. How things work: Wisdom teeth removal. Available at: https://www.tiktok.com/@how.thingss. workk/video/6975911053792267526?lang=en\&is_copy_ url=1\&is_from_webapp=v1 (accessed September 2021).

8. TikTok. How porcelain veneers are glued on. Available at: https://www.tiktok.com/@drtejaspatel/ video/6943409231097761029?lang=en\&is_copy_ url=1\&is_from_webapp=v1 (accessed September 2021).

9. TikTok. Making retainers like a swagger. Available at: https://www.tiktok.com/@chris.nge/ video/6903722853816929538?lang=en\&is copy_url=1\&is_from_webapp=v1 (accessed September 2021). 